\title{
Determination of Broadening Functions using the Singular Value Decomposition (SVD) Technique
}

\author{
Slavek Rucinski \\ Canada-France-Hawaii Telescope Co., Kamuela, HI 96743, USA
}

\begin{abstract}
The cross-correlation function (CCF) has become the standard tool for extraction of radial-velocity and broadening information from high resolution spectra. It permits integration of information which is common to many spectral lines into one function which is easy to calculate, visualize and interpret. However, the CCF is not the best tool for many applications where it should be replaced by the proper broadening function $(\mathrm{BF})$. Typical applications requiring use of BFs rather than CCFs involve finding locations of star spots, studies of projected shapes of highly distorted stars such as contact binaries (as no assumptions can be made about BF symmetry or even continuity) and $[\mathrm{Fe} / \mathrm{H}]$ metallicity determinations (good baselines and avoidance of negative lobes are essential). It is stressed that the CCFs are not broadening functions. This note concentrates on the advantages of determining BFs through the process of linear inversion, preferably accomplished using the singular value decomposition (SVD). Some basic examples of numerical operations are given in the IDL programming language.
\end{abstract}

\section{Convolution and cross-correlation}

Convolution is an operation that nature does for us. We seldom see "naked" functions. These could be the convolution of a spectrum with the spectrograph's instrumental profile or with the radial component of the micro-turbulence velocity field in the stellar atmosphere or with a broadening function due to rapid rotation of a star. Thus, instead of a function $f(u)$, we observe a function $h(x)$ which is a convolution with some other broadening function (BF), $g(x)$ :

$$
h(x)=\int_{-\infty}^{+\infty} f(u) g(x-u) d u=f(x) * g(x)
$$

This natural process can be easily simulated in numerical packages (examples in the IDL programming language are marked by a command-line prompt IDL $>$ ) either by a special operator:

IDL $h=\operatorname{convol}(f, g)$

or through the Fourier-transform multiplication and its inverse:

IDL $>h=$ float $(\mathrm{fft}(\mathrm{fft}(\mathrm{f},-1) * f f t(g,-1),+1))$

Cross-correlation is an operation which for real functions differs from the convolution really only in the symmetry of the arguments. For complex functions things are slightly different (real and imaginary parts have different symmetries), 
but astronomers observe real spectra so we do not have to worry about the mathematical nuances.

$$
c(x)=\int_{-\infty}^{+\infty} f(u) g(u+x) d u=f(x) \star g(x)
$$

The cross-correlation (note the different asterisk above) function can be computed numerically using:

IDL> lag = findgen (201) -100

IDL> c = c_correlate $(f, g, l a g)$

\section{Broadening functions}

Suppose we observe a sharp-line spectrum $(S(\lambda))$ and a broad-line spectrum $(P(\lambda))$ and we want to determine the broadening and any other differences which make the latter spectrum more interesting than the former. The function $B(\lambda)$ can be a rotational broadening function for a single, rapidly-rotating star, or a more complex profile for two components of a binary, or for a star with spots (where they would show as indentations in the function). The sharpline spectrum $S(\lambda)$ is not free of some broadening. This can be the thermal broadening of lines or micro-turbulence or some other mechanisms; we call them jointly $T(\lambda)$. Thus, schematically, the sharp-line spectrum can be written as:

$$
S(\lambda)=\left(\sum_{i} a_{i} \delta\left(\lambda_{i}\right)\right) * T(\lambda)
$$

while the broad-line spectrum, broadened additionally by $B(\lambda)$, can be written as:

$$
P(\lambda)=S(\lambda) * B(\lambda)=\left(\sum_{i} a_{i} \delta\left(\lambda_{i}\right)\right) * T(\lambda) * B(\lambda)
$$

The cross-correlation (CCF) with the sharp-line spectrum is frequently taken as an estimate of $B(\lambda)$ :

$$
C(\lambda)=S(\lambda) \star P(\lambda)=S(\lambda) \star(S(\lambda) * B(\lambda))=T(\lambda) * T(\lambda) * B(\lambda)
$$

The new function $\mathcal{B}(\lambda)$, is not identical to $B(\lambda)$, because it inherits the common broadening components (such as thermal, micro-turbulence, instrumental) from both spectra. Tonry \& Davis (1979) showed that if those common components are represented by gaussians, the addition is quadratic, which for these functions means repeated convolutions. Thus, the CCF cannot really be used to replace the broadening function. But it can give us some approximation of it and will remain a useful tool to have some preliminary estimate of the degree of line broadening. For symmetrical broadening functions, it will remain the simplest tool to determine the radial velocities simultaneously from many spectral lines.

The differences between the BF and the CCF can be seen when an artificially broadened spectrum is created by a convolution and then the resulting spectrum is subjected to the CCF operation. The result (Figure 1) is obviously different from the BF: The CCF shows negative baseline excursions and, most worryingly, it shows the "peak-pulling" effect which would lead to an under-estimate of the individual component velocities. While this last problem can be overcome by applying the TODCOR technique (Zucker \& Mazeh 1994), we clearly see that the CCF is not the BF. 

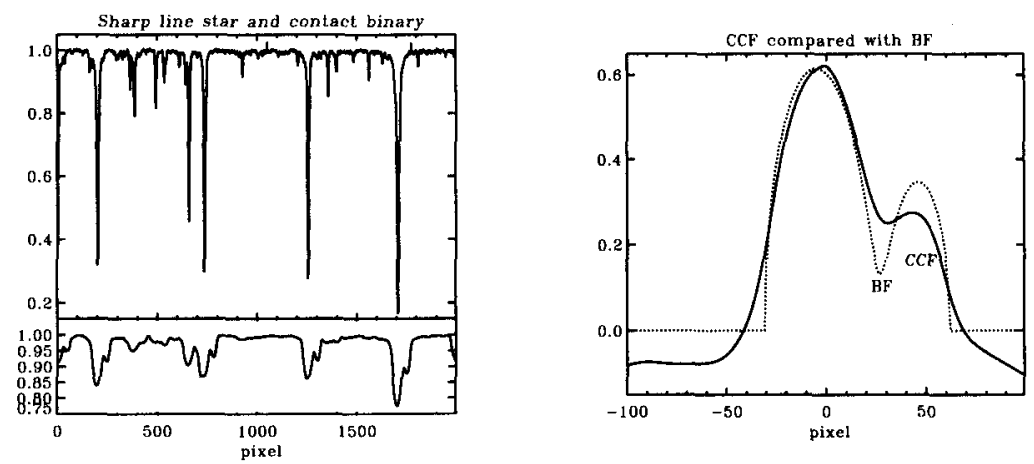

Figure 1. The left panel shows a sharp-line spectrum (upper part) and the result of convolving it with the broadening function for a contact binary (lower part); this function is shown in the right panel by the dotted line $(\mathrm{BF})$. The cross correlation of the broadened spectrum (with added noise to have $S / N=100$ ) with the sharp spectrum is shown in the right panel by the continuous line. Notice the negative baseline and the peak-pulling in the CCF.

\section{The Fourier transform de-convolution}

Some attempts to determine the broadening functions (Anderson et al. 1983) utilized the well known property of Fourier transforms of a correspondence between convolutions and multiplications in the two relevant domains. Thus, a convolution:

$$
P(\lambda)=S(\lambda) * B(\lambda)
$$

transformed with the Fourier transform $\mathcal{F}$ changes into a product of the transforms:

$$
\mathcal{F}\{P(\lambda)\}=\mathcal{F}\{S(\lambda)\} \cdot \mathcal{F}\{B(\lambda)\}
$$

Therefore, the broadening function can be restored with:

$$
B(\lambda) \simeq \mathcal{F}^{-1}\{\mathcal{F}\{P(\lambda)\} / \mathcal{F}\{S(\lambda)\}\}
$$

This can be done in a compact way with:

IDL $>b=$ float (fft $(\mathrm{fft}(\mathrm{p},-1) / \mathrm{fft}(\mathrm{s},-1),+1))$

While the mathematical background is simple and easy, the practice is just the opposite. First, the resulting $B(\lambda)$ spans the whole spectral window, so that one determines a lot of zeroes; there is no "compression" information whatsoever. But, more importantly, the division operation simply does not work: the high frequency noise becomes amplified and some sort of frequency filtering is needed. The result may then actually depend on the applied filter.

Some authors (see for example the large collection of works of D. F. Gray) use spectra transformed into the frequency domain without the division step. This can be done for broadening mechanisms which can be described by simple functions or obeying some symmetries, but fails in cases of spots or of BFs of close binary systems. 


\section{Convolution in the formalism of linear equations}

There are two main issues that re-casting the convolution into a set of linear equations can resolve. These are: (1) How to channel information over the whole spectrum (say 2000 pixel long) into the BF window (say 200 pixels long)? (2) How to utilize all the information contained in sharp-line spectra and remove the influence of the noise in the continuum (which carries no information)?

The convolution can be written as an over-determined system of linear equations which link a sharp-line spectrum $\vec{S}(n)$, via the broadening function $\vec{B}(m)$, with the broadened spectrum $\vec{P}(m)$. The mapping is through the "design matrix" $\widehat{D e s}(m, n)$ which is formed from the sharp line spectrum $\vec{S}(n)$ by consecutive vertical shifts by one element. In IDL, this can be done with a simple routine:

function map4, s, m

; $m$ - must be odd, $n$ must be even

$\mathrm{n}=\mathrm{n}_{-} e l$ ements $(s) \& \mathrm{t}=\mathrm{fltarr}(\mathrm{m}) \# \operatorname{fltarr}(\mathrm{n}-\mathrm{m}+1)$

; $t(m, n-m)=t($ small, large-small) dimensions

for $j=0, m-1$ do for $i=m / 2, n-m / 2-1$ do $t(j, i-m / 2)=s(i-j+m / 2)$

return, $t$

end

An example of using this routine to create a design matrix for a 201-pixel long window would be:

IDL> des $=\operatorname{map} 4(s, 201)$.

The program spectra must accordingly be trimmed to $n-m+1$ with:

IDL> $p=p(m / 2: n-m / 2-1)$.

The system of equations has a familiar form of the over-determined linear set:

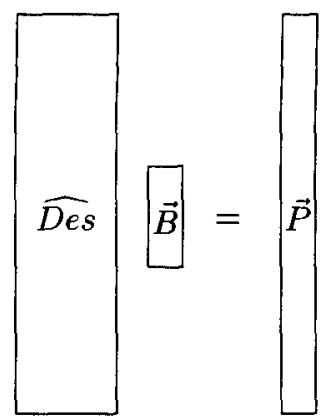

\section{Singular value decomposition (SVD)}

One of the traditional ways of solving the system of equations above would be to transform it into a system of the "normal" equations of the size reduced from $m \times(n-m)$ to $m \times m$ by multiplication of both sides by the transpose of the design matrix. The result would be the BF defined in the least-squares sense, and it is possible to stop at this point. However, the singular value decomposition technique also gives us such an answer, but - in addition - makes it possible to remove the influence of the continuum and its noise. 
The SVD technique is beautifully described in the "numerical techniques Bible" of Press et al. (1986). They present it as a somewhat magic black box and for most users it is just fine. If you want to learn how the technique really works, then the books of Golub \& Van Loan (1989) or Craig \& Brown (1986) are probably the best references.

The essence of the SVD is the property that one can represent any matrix by a product of 3 matrices; in our case: $\widehat{D e s}=\hat{U} \widehat{W} \hat{V}^{T}$. These matrices are: the column ortho-normal $\widehat{U}$ and $\widehat{V}$ and the diagonal matrix $\widehat{W}$ (this is really a vector containing the diagonal elements). The property of the columns in $\widehat{U}$ and $\widehat{V}^{T}$ is that the following products, $\widehat{U}^{T} \widehat{U}=\widehat{I}$ and $\widehat{V}^{T} \widehat{V}=\widehat{I}$, give the unity array $\widehat{I}$ (1 on the diagonal).

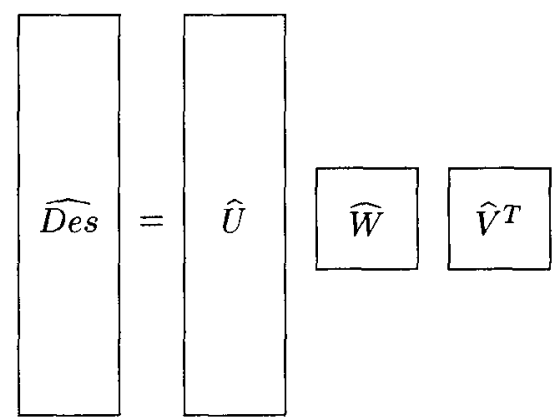

In IDL, the operation is represented by: IDL $>$ svdc, des, $w, u, v, /$ double. Here, des is the only input quantity and the remaining parameters are what the routine produces as output. The keyword /double is for higher precision and is optional.

One can check the correctness of the operations by the following commands: IDL $>$ wf $=f l \operatorname{tarr}(m, m)$

IDL $>$ for $i=0, m-1$ do $w f(i, i)=w(i)$

IDL > des_check $=u$ \#\# wf \#\# transpose $(v)$

The three new matrices are all invertible: $\widehat{U}$ and $\widehat{V}$ are ortho-normal arrays, so that their inverses are just transposes, while the diagonal array $\widehat{W}$ is replaced by a diagonal array $\widehat{W 1}$, with the diagonal elements containing the inverses, $w 1_{i, i}=1 / w_{i}$ :

IDL> พ1 $=f I \operatorname{tarr}(m, m)$

IDL> for $i=0, m-1$ do $w 1(i, i)=1 . / w(i)$

The solution is given by: $\vec{B}=\widehat{V} \widehat{W_{1}}\left(\hat{U}^{T} \vec{P}\right)$ or schematically: 


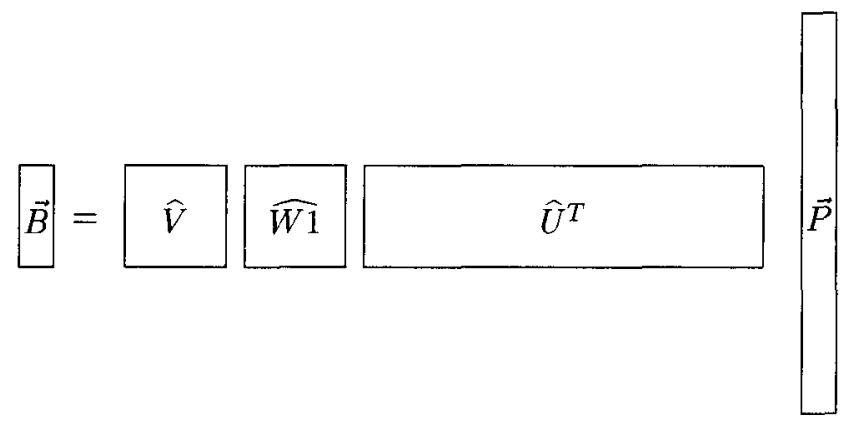

Numerically:

IDL> $b=\operatorname{reform}(v \# \# w 1 \#$ (transpose(u)\#\#)).

A routine makes this simpler:

IDL $>\mathrm{b}=\operatorname{svsol}(\mathrm{u}, \mathrm{w}, \mathrm{v}, \mathrm{p}, /$ double $)$.

The elements of $\vec{B}$ are all independent, so that any - even strange or discontinuous - broadening functions can be restored as no condition imposed on the smoothness or symmetry of the result. Note that, if only one sharp-line template is used, the decomposition operation svdc is done only once, for possibly many broad-line spectra $\mathrm{p}$, each giving a separate solution $\mathrm{b}$.

\section{Advantages and disadvantages of the SVD approach}

On the positive side: (1) The problem can be treated as a set of linear equations. (2) An "inverse" of the rectangular array $\widehat{D e s}$ is possible. (3) The solution of $\vec{B}$ is defined in the least-squares sense (shortest modulus). (4) The result is the real broadening function. But there are also minuses: (5) One must solve a large system of, say, 2000 equations for 200 unknowns. (6) One must know a priori how many unknowns. (7) Initially, the results may turn out quite poorly, because of the presence of plenty of linearly-dependent equations in the system (parts of the spectra where the featureless continuum provides no broadening information).

The SVD approach offers a simple resolution of (7) as it permits removal of the effects of the continuum in an objective way. The key element here is the singular values contained in the diagonal of $\widehat{W}$. Since the solution involves $1 / w_{i}$, small values in $w_{i}$ spoil the solution. These are exactly those problematic values that one wants to avoid. Thus, by rejecting small values of $w_{i}$, one can (i) remove the linearly dependent equations, (ii) diminish the influence of the noise from the continuum, (iii) reduce the influence of the computer round-off errors (which enter multiplied by the order of the problem) and (iv) reduce the number of unknowns (because the system is usually not over-determined at all). All these properties are related to the "conditioning" of the array $\widehat{D e s}$. The reader is directed to the source texts on this subject for further reading.

The important factor is $\max \left(w_{i}\right) / \min \left(w_{i}\right)$ which provides an estimate on how many of the singular values should be used. In practice, one can keep on adding more $w_{i}$ and see the successively better solutions. The diagonal arrays $\widehat{W}$ and $\widehat{W 1}$ will then have elements: $w_{i}=w_{0}, w_{1}, w_{2}, w_{k}, \ldots, w_{m-1}$ and $w 1_{i}=$ 

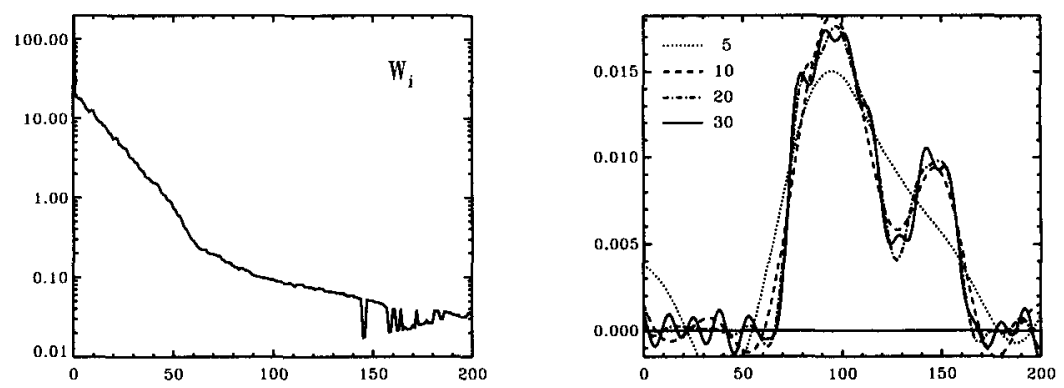

Figure 2. The left panel shows the run of the singular values for the sharp-line spectrum in Figure 1. The right panel gives the solutions for the broad-line spectrum in the same figure (with added noise at $S / N=100$ ) utilizing the first $5,10,20$ and 30 singular values.

$1 / w_{0}, 1 / w_{1}, 1 / w_{k-1}, 0, \ldots, 0$ with $k$ (we call it the order of solution) spanning the whole range 0 to $m-1$. In IDL, this can be done by forming a square matrix of solutions:

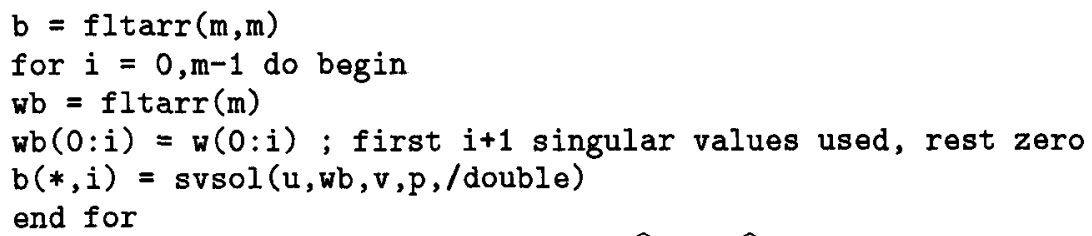

We note in passing, that the arrays $\widehat{U}$ and $\hat{V}$ have very special properties as they contain the basis vectors in the spaces of the spectra and broadening functions, respectively. One can see this by analyzing a diagonalized system: $\widehat{W} \vec{Z}=\vec{D}$, obtained by keeping the same $\widehat{W}$, with $\vec{Z}=\hat{V}^{T} \vec{B}$ and $\vec{D}=\widehat{U}^{T} \vec{P}$. The solution of the diagonalized system would then be: $\vec{Z}=\vec{D} / \vec{W}$, but, in practice, the diagonal of $\widehat{W}$ is a vector, so that $z_{i}=d_{i} / w_{i}$. Plotting the columns of $\widehat{U}$ and $\widehat{V}$ can tell one a lot about the conditioning of the solution.

\section{A few notes on the SVD solutions}

The first question is: How far in $k$ should one go and where to stop? The essential operation is to plot (usually in $\log$ units) the vector $\vec{W}$ (Figure 2). There are 3 parts of it: (1) the good, large singular values, (2) the small values usually representing the noise in the spectrum $S(\lambda)$ and (3) the numerical errors. You may want to stop no later than at the kink below the good part. But the real "quality control" is the fit to $\vec{P}$. If the error of the fit stops decreasing, you have found the right point (Figure 3). Beyond that point, you will start fitting the noise! However, it is useful to analyze the solutions for different orders $k$ and see how they first improve and then get worse. Sometimes the fit will remain poor, in spite of the leveling of the error curve; this usually means a wrong choice of the sharp-line spectrum. The standard error of the fit can be calculated from: sig $=f 1 \operatorname{tarr}(m)$; error 

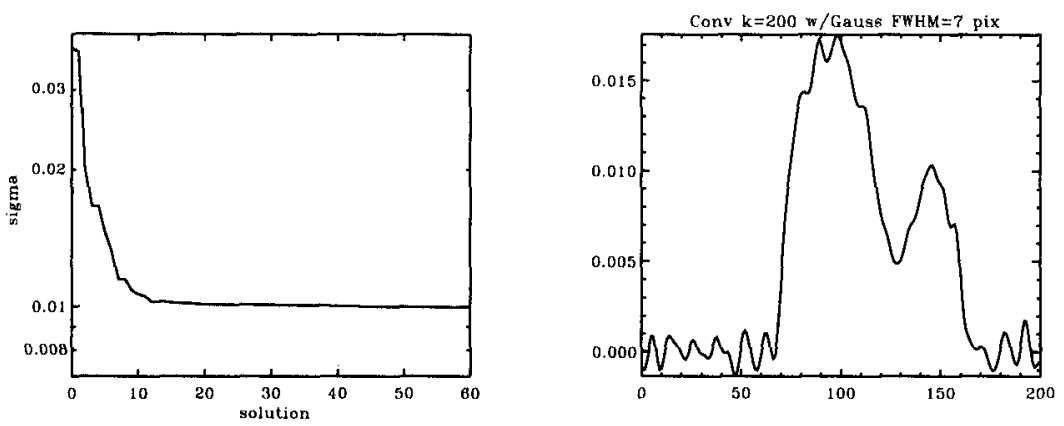

Figure 3. The left panel shows the mean standard error of the fit for our example solutions utilizing progressively more singular values. It is obvious that the first $10-15$ singular values give an adequate fit (compare with Figure 2). The right panel shows the solution utilizing all 200 singular values, convolved with a gaussian. This approach may offer a better control over the final resolution of the BF.

\section{pred = des \#\# transpose(b) ; predicted fits}

for $i=0, m-1$ do $\operatorname{sig}(i)=\operatorname{sqrt}(\operatorname{total}((\operatorname{pred}(i, *)-p)-2) / m)$

Usually, even very low order solutions are well defined (Figure 2), but stopping early is not always advisable because this leads to a loss of resolution, as then not all basis vectors contribute. The solution which - in principle - has all elements in $\vec{B}$ independent suddenly acquires inter-element correlations. Thus, it may be advantageous to go to a highest possible order $(k=m)$ and thus insure that elements of $\vec{B}$ are uncorrelated, and then decrease the noise by smoothing.

One should be aware that the errors may be under-estimated for the case of truncated $(k<m)$ solutions. While the prescriptions of Rix \& White (1992) and Rucinski, Lu \& Shi (1993) are based on the theory of the full SVD, the error analysis for the truncated case has not yet been done. In this situation, it may be advantageous to utilize techniques of the external estimates, such as the bootstrap or Monte Carlo. This subject certainly requires more work.

\section{Conclusions}

One can position the SVD technique of linear broadening function restoration as located between the cross-correlation and the direct modeling of the spectra. The BFs determined with it are much better defined than the CCFs, and are true broadening functions, not their proxies. They also integrate the geometrical information from a spectrum, but give well defined baselines without the CCF's negative fringes. They do require a bit more computer work, but several numerical packages can easily handle large linear systems of equations involved here. Obviously, they cannot replace the spectrum synthesis, if these are needed, but can be a useful tool in their preparation. 


\section{Discussion}

Cameron: Would you like to comment on the effect of using delta functions, rather than a template spectrum, on the stability of the result?

In practice, we find that even though there are inevitably errors in the strengths and positions of the delta functions, provided that the reference spectra and target spectra are deconvolved with the same mask, the results are both stable against wiggles and internally self-consistent.

Rucinski: If the template spectrum consists of $\delta$-functions, then the difference between the cross-correlation and linear decomposition basically disappears. In fact, a "good" forest of $\delta$-functions should give better results than an observatioonal sharp-line spectrum. I like your approach based on the use of the $\delta$-functions!

Kürster: SVD does not impose a positivity constraint on the solution as other methods, such as maximum entropy, do. Could you comment on why you prefer SVD nevertheless?

Rucinski: One of the possibilities is to use the property of non-negativity for determining how many singular values should be included in the solution. This property was used before to find the plasma emission measures from EUV spectra (by Schmidt et al.) and can be used in the BF determinations as well.

\section{References}

Anderson, L., Stanford, D. \& Leininger, D. 1983, ApJ, 270, 200

Craig, I.J.D. \& Brown, J.C. 1986, Inverse Problems in Astronomy, (Bristol and Boston: Adam Hilger Ltd)

Golub, G.H. \& Van Loan, C.F. 1989, Matrix Computations, 2nd ed. (Baltimore: Johns Hopkins Univ. Press)

Press, W.H., Teukolsky, S.A., Vetterling, W.T. \& Flannery, B.P. 1986, Numerical Recipes, Cambridge University Press (all editions)

Rix, H.-W. \& White, S.D.M. 1992, MNRAS, 254, 384

Rucinski, S.M. 1992, AJ, 104, 1968

Rucinski, S.M., Lu, W.-X. \& Shi, J. 1993, AJ, 106, 1174;

Tonry, J. \& Davis, M. 1979, AJ, 84, 1511

Zucker, S. \& Mazeh, T. 1994, ApJ, 420, 806 